\title{
Service outcome of antiretroviral Post-Exposure Prophylaxis (PEP) for occupational HIV exposure among health care personnel
}

Asha Shah, Burzin Kavina, Sanjeev Prajapati, Hemang Purohit, Manoj Shevkani*, Urvi Derasari, Umesh Nihalani, Bipin Amin, Bankim Mankad, Girish Prajapati

From $16^{\text {th }}$ International Symposium on HIV and Emerging Infectious Diseases Marseille, France. 24-26 March 2010

\section{Background}

This study aims to assessment of the outcome of Post Exposure Prophylaxis (PEP) Service at Centre of Excellence (CoE), ART Centre, B. J. Medical College, Civil Hospital, Ahmedabad, Gujarat, India among Health Care Personnel (HCP).

\section{Methods}

Potentially exposed to infectious materials HCPs of the institute were studied. Exposed HCP were enrolled and followed up as per Management of Occupational Exposure including PEP for HIV by National AIDS Control Organization (NACO) India guidelines.

\section{Results}

Total of 43 exposed HCP (18 male and 25 female with male to female ratio 0.72: 1) during October 2008 to October 2009 were studied, Table 1.

\section{Discussion}

Those tested for HIV were all Non - Reactive; shows the effectiveness of PEP services. We are finding obstacles on follow up tracking like "I am much aware about the risk, stigma of HIV Positivity, negligence for follow up and confidentiality issues at the workplace etc." that prevent the HCP for PEP and increase the risk of transmission. Practice and regular sensitization with UPW has increased the awareness among HCP for reducing HIV infection risk and transmission and prevention through PEP.

\footnotetext{
* Correspondence: coe.art.ahmedabad@gmail.com

ART center B J Medical College, Ahmedabad, India
}

Published: 11 May 2010

doi:10.1186/1742-4690-7-S1-P75

Cite this article as: Shah et al:: Service outcome of antiretroviral PostExposure Prophylaxis (PEP) for occupational HIV exposure among health care personnel. Retrovirology 2010 7(Suppl 1):P75.
Submit your next manuscript to BioMed Central and take full advantage of:

- Convenient online submission

- Thorough peer review

- No space constraints or color figure charges

- Immediate publication on acceptance

- Inclusion in PubMed, CAS, Scopus and Google Scholar

- Research which is freely available for redistribution

Submit your manuscript at www.biomedcentral.com/submit
C Biomed Central 
Table 1

\begin{tabular}{|c|c|c|}
\hline \multicolumn{2}{|l|}{ Parameter } & $\begin{array}{c}\text { No. Of Cases } \\
(n=43)\end{array}$ \\
\hline \multicolumn{3}{|c|}{ Universal Precaution Workshop (UPW) for HCP } \\
\hline \multicolumn{2}{|l|}{ January 2008 - December 2008} & 2 \\
\hline \multicolumn{2}{|l|}{ January 2009 - October 2009} & 9 \\
\hline \multicolumn{3}{|c|}{ Enrolment of HCP for PEP at CoE } \\
\hline \multicolumn{2}{|l|}{ October 2008 - May 2009} & $19(44.18 \%)$ \\
\hline \multicolumn{2}{|l|}{ June 2009 - October 2009} & $24(55.81 \%)$ \\
\hline \multicolumn{3}{|c|}{ HCP Category Enrolled for PEP at CoE } \\
\hline \multicolumn{2}{|l|}{ Medics } & $19(44.18 \%)$ \\
\hline \multicolumn{2}{|l|}{ Paramedics } & $21(48.8 \%)$ \\
\hline \multicolumn{2}{|l|}{ Servants } & $3(7 \%)$ \\
\hline \multicolumn{3}{|c|}{ HIV Status of the HCP for PEP enrolment } \\
\hline \multirow[t]{3}{*}{ October 2008-May 2009(n = 19) } & Reactive & 0 \\
\hline & Non - Reactive & $7(36.8 \%)$ \\
\hline & Unknown & $12(63.15 \%)$ \\
\hline \multirow[t]{3}{*}{ June 2009-October 2009(n = 24) } & Reactive & 0 \\
\hline & Non - Reactive & $16(66.66 \%)$ \\
\hline & Unknown & $3(12.5 \%)$ \\
\hline \multicolumn{3}{|c|}{ Type of Exposure } \\
\hline \multicolumn{2}{|l|}{ Mild } & $21(48.8 \%)$ \\
\hline \multicolumn{2}{|l|}{ Moderate } & 18(41.9\%) \\
\hline \multicolumn{2}{|l|}{ Severe } & $4(9.3 \%)$ \\
\hline \multicolumn{3}{|c|}{ Reporting Timeline of exposed HCP to the CoE } \\
\hline \multicolumn{2}{|l|}{$<2$ hours } & $27(62.8 \%)$ \\
\hline \multicolumn{2}{|l|}{$2-24$ hours } & $9(20.9 \%)$ \\
\hline \multicolumn{2}{|l|}{$24-72$ hours } & $5(11.6 \%)$ \\
\hline \multicolumn{2}{|l|}{$>72$ hours } & $2(4.7 \%)$ \\
\hline \multicolumn{3}{|c|}{ HIV Status of the source } \\
\hline \multicolumn{2}{|l|}{ Reactive } & $22(51.2 \%)$ \\
\hline \multicolumn{2}{|l|}{ Unknown } & $21(48.8 \%)$ \\
\hline \multicolumn{3}{|c|}{ PEP Regimen Prescribed } \\
\hline \multicolumn{2}{|l|}{ Basic (Zidovudine+Lamivudine) } & $30(69.76 \%)$ \\
\hline Expanded (Zidovudine+Lamivudin & Lopinavir/Ritonavir) & $12(27.90 \%)$ \\
\hline Expanded (Zidovudine+Lamivudin & Indinavir) & $1(2.32 \%)$ \\
\hline $\begin{array}{l}\text { HIV Status of exposed HCP po } \\
\qquad(n=18)\end{array}$ & PEP at 6 months & \\
\hline Reactive & & 0 \\
\hline Non - Reactive & & $3(16.66 \%)$ \\
\hline Unknown & & $15(83.33 \%)$ \\
\hline HCP Not Completed $6 \mathrm{Mc}$ & hs $(n=43)$ & $25(58.13 \%)$ \\
\hline
\end{tabular}

